\title{
Redução das concentrações de cloreto de sódio na alimentação visando a homeostase da pressão arterial
}

Reduction of concentrations of sodium chloride in food aiming blood pressure homeostasis

\author{
Anderson Martelli ${ }^{1}$ \\ ' Faculdade Mogiana do Estado de São Paulo, Mogi Guaçu, SP, Brasil
}

\section{Resumo}

\begin{abstract}
A hipertensão arterial (HA) é uma condição clínica multifatorial caracterizada por níveis elevados de pressão arterial. A ingestão excessiva de sódio, mineral frequentemente presente nos alimentos, tem sido correlacionada com a HA. Esta revisão apresenta como objetivo descrever a relação entre o consumo excessivo de sódio e o desenvolvimento e/ou agravo da HA e como sua redução na alimentação pode controlar os níveis da pressão arterial. A pesquisa foi realizada a partir de uma revisão bibliográfica da literatura especializada, sendo consultado artigos científicos publicados até 2012 e adicionalmente consulta de livros acadêmicos para complementação das informações. Estudos baseados em inquéritos alimentares demonstram que a população brasileira apresenta elevada ingestão de sódio o qual provem principalmente do sal de cozinha e de condimentos com este ingrediente, embora apontem também que há um aumento linear e intenso do total de sódio proveniente de alimentos processados, sendo descrito em vários estudos que a ingestão excessiva de sódio eleva a pressão arterial potencializando o desenvolvimento de outras doenças cardiovasculares.
\end{abstract}

Palavras-chave: Sódio; hipertensão arterial; dieta; fatores de risco

\begin{abstract}
Arterial hypertension (AH) is a multifactorial clinical condition characterized by high levels of blood pressure. Excessive intake of sodium, mineral often present in food, has been correlated with the HA. This review aims to describe the relationship between excessive consumption of sodium and the development and/or aggravation of hypertension and how their reduction in the diet can control blood pressure levels. The survey was conducted from a literature review of the literature, consulted with scientific articles published by 2013 and further consultation with academic books to complement the information. Studies based on dietary surveys show that the Brazilian population has a high sodium intake which stems primarily of salt and condiments with this ingredient, but also indicate that there is a linear increase of total heavy and sodium coming from processed foods, been described in several studies that excessive sodium intake increases blood pressure enhancing the development of other cardiovascular diseases.
\end{abstract}

Keywords: Sodium, hypertension, diet, risk factors 


\section{INTRODUÇÃO}

É consensual o papel da alimentação e nutrição como fator determinante nas doenças crônicas não transmissíveis, sendo-lhes dado bastante atenção na prevenção de diversas patologias. No contexto atual, na qual as doenças crônicas não transmissíveis assumem uma importância cada vez maior, mesmo nos países subdesenvolvidos, em relação às doenças contagiosas, parece-nos, cada vez mais relevante, considerar os perigos de caráter nutricional.

Nas últimas décadas, o consumo de sódio através da alimentação na maioria dos países tem sido excessivo, variando de 9 a 12 g por pessoa/ dia (BROWN et al., 2009). Segundo Salas (2009), o Brasil está classificado entre os maiores consumidores mundiais de sal, com média de ingestão de 15,09 gramas diários.

Em contraste, a Organização Mundial da Saúde (OMS) recomenda uma ingestão diária, para adultos, de no máximo $5 \mathrm{~g}$ de sal (equivalentes a $2000 \mathrm{mg}$ de sódio). Para crianças e adolescentes, os limites máximos do consumo de sódio e sal são ainda menores, visto serem populações mais vulneráveis (NILSON et al., 2012). Segundo Bibbins et al. (2010), a redução do consumo desse mineral nessas faixas etárias precoces representa melhoria da saúde cardíaca na vida adulta.

Também existem evidências em modelos animais, de que o consumo de sódio na gravidez pode determinar um aumento da preferência por sódio na fase adulta nos descendentes (NICOLAIDIS, 2008) indicando a necessidade de maior atenção também entre as gestantes (NILSON et al., 2012).

Há cerca de 5000 anos atrás, Huang Ti Nei Ching Su Wenn, um médico chinês, descreveu uma doença relacionada com o consumo indiscriminado de sal e em paralelo o sódio. No entanto, o primeiro trabalho científico que estabeleceu uma relação linear entre o consumo de sal e pressão arterial foi publicado muito anos mais tarde, por Dalh em 1960 (VIEGAS, 2008).

Atualmente, esta bem estabelecido a associação entre o consumo excessivo de sódio e o desenvolvimento de doenças crônicas não transmissíveis, desde a HA e doenças cardiovasculares até o câncer de estômago, doenças renais e osteoporose, entre outras (HE; MACGREGOR, 2007). A HA está associada a altos índices de morbimortalidade e constitui um dos grandes problemas de saúde pública no mundo, dada a sua alta prevalência (RIELLA; MARTINS, 2001; DRAGER; KRIEGER, 2004).

No Brasil, as doenças cardiovasculares são responsáveis por mais de 250.000 mortes por ano, a hipertensão arterial sistêmica (HAS) participa de quase metade delas (MIRANDA et al., 2002). A HAS é uma situação clínica de natureza multifatorial caracterizada por níveis de pressão arterial elevados. De acordo com Waitzberg (2001), as alterações dos níveis pressóricos podem ser decorrentes de fatores genéticos e ambientais. $\mathrm{O}$ fator genético é atribuível à genética comum de base (herança familiar) e os fatores ambientais atuam nos indivíduos suscetíveis, por ambiente compartilhado ou hábitos de estilo de vida, como o excesso na ingestão de sódio.

McArdle et al (2011), relatam que 50\% dos pacientes hipertensos procuram de fato algum tipo de tratamento e uma das razões dessa falta de complacência diz respeito aos possíveis efeitos colaterais da medicação anti-hipertensiva como a fadiga e impotência fazendo com que os pacientes não consigam manter um esquema crônico de medicamentos.

Assim, as ações voltadas à redução do consumo de sódio se destacam entre as ações de prevenção e controle das doenças crônicas diretamente associadas à alimentação por uma relação positiva entre custo e efetividade (WANG; LABARTHE, 2011; WEBSTER et al., 2011). Entre as principais estratégias encontram-se a redução voluntária do conteúdo de sódio de alimentos processados e a realização de campanhas de mídia para a promoção de hábitos alimentares saudáveis, que, segundo estimativas da OMS, poderiam evitar 2,5 milhões de mortes e poupar bilhões de dólares aos sistemas de saúde no mundo (NILSON et al., 2012).

No Brasil, o Ministério da Saúde tem coordenado estratégias nacionais com vistas à redução do consumo de sódio, através de ações articuladas a planos setoriais como o Plano Nacional de Saúde 2012-2015 e o Plano de Ações Estratégicas para o Enfrentamento das Doenças Crônicas Não-Transmissíveis no Brasil 2011-2022 (NILSON et al., 2012).

Se tratando das doenças crônicas, a HA, uma das principais patologias relacionadas ao consumo de sódio com grande importância epidemiológica no Brasil. Dados da Vigilância de Fatores de Risco e Proteção para Doenças Crônicas por Inquérito Telefônico (Vigitel) do Ministério da Saúde apontam 
que $23,3 \%$ da população adulta residente nas capitais brasileiras referiram diagnóstico médico de HA em 2010 (MINISTÉRIO DA SAÚDE, 2010).

Estudos populacionais e epidemiológicos descritos na literatura especializada sustentam que o sódio tem seu papel na etiologia da HA, assim, levando em consideração o elevado consumo de sódio pela população brasileira, sua influência na prevalência de HA e a importância de controlar este consumo, o presente estudo teve como objetivo realizar uma revisão bibliográfica estruturada sobre o consumo excessivo de sódio na alimentação e o desenvolvimento da HA, e como efeito paradoxal, a sua restrição visando a homeostase da pressão arterial.

\section{DESENVOLVIMENTO}

Para a composição da presente revisão foi realizado um levantamento bibliográfico entre os meses de outubro a dezembro de 2013 nas bases de dados Medline, Scielo, Lilacs, Portal de Periódicos da Coordenação de Aperfeiçoamento de Pessoal de Nível Superior (CAPES) e a busca de dados no Google Acadêmico de artigos científicos publicados até 2012 utilizando como descritores isolados ou em combinação: sódio, hipertensão arterial, dieta e doenças cardiovasculares, sendo realizado adicionalmente a consulta de livros acadêmicos para complementação das informações sobre a ingestão de sódio e a fisiopatologia da HA.

Para seleção do material, efetuaram-se três etapas. A primeira foi caracterizada pela pesquisa do material utilizado na revisão. A segunda compreendeu a leitura dos títulos e resumos desses artigos, visando uma maior aproximação e conhecimento, sendo excluídos os que não tivessem relação e relevância com o tema. Após essa seleção, buscaram-se os textos que se encontravam disponíveis na íntegra sendo estes, inclusos na revisão figura 1.

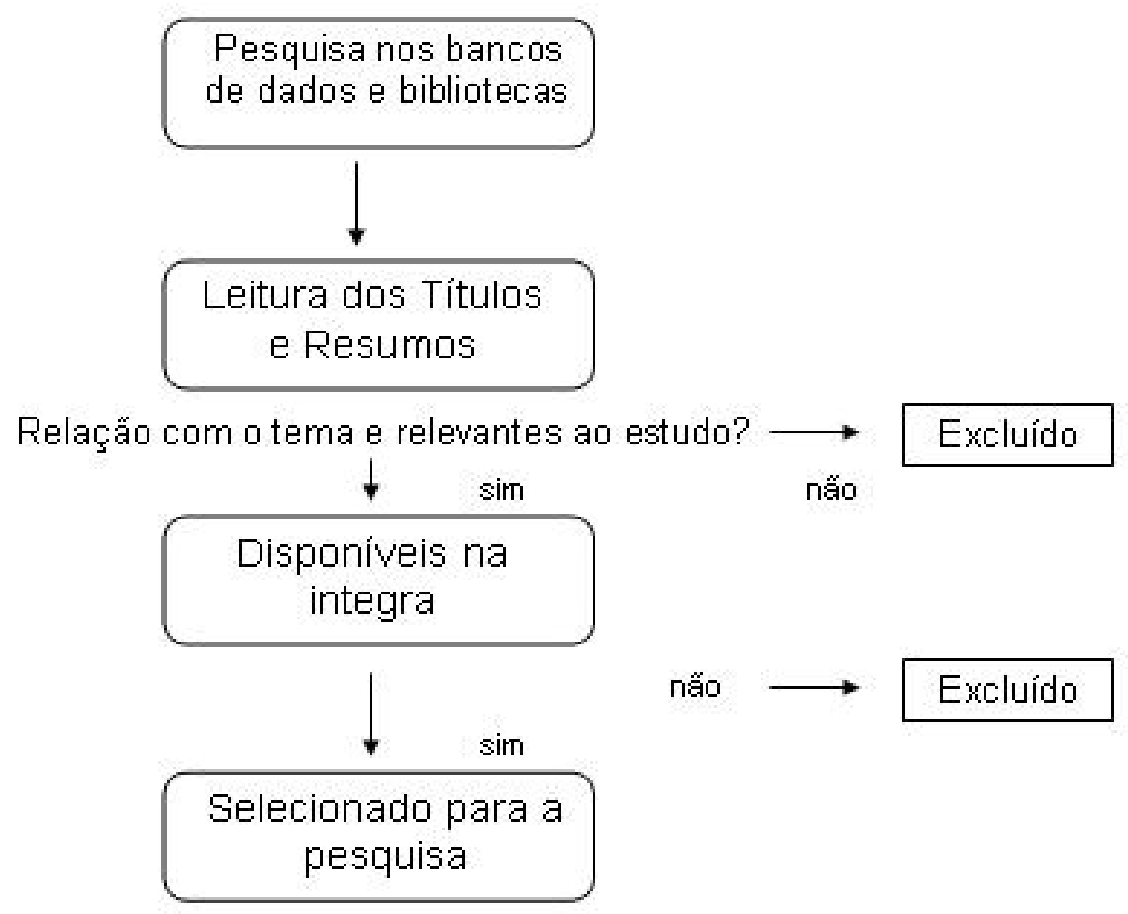

Figura 1. Fluxograma relativo às etapas de seleção dos artigos utilizados na pesquisa

Dos artigos selecionados e incluídos na pesquisa constituíram ensaios clínicos, artigos originais, revisões e revisões sistemáticas. Como critérios de elegibilidade e inclusão dos artigos, analisaram-se a procedência e indexação das revistas, estudos que apresentassem dados referentes ao consumo de sódio na alimentação e a evolução do estabelecimento dos quadros de HA preconizado entre os anos de 1996 até o mais atual 2012. Na leitura e avaliação, os artigos que apresentaram os critérios de elegibilidade foram selecionados e incluídos na pesquisa por consenso. 


\section{SÓDIO E PRESSÃO ARTERIAL}

A HA possui duas classificações, considerando-se a hipertensão primária (ou essencial), representando 90\% dos casos e, cuja causa é desconhecida, e a hipertensão secundária, a qual está sempre subjacente a uma doença, como por exemplo, uma patologia renal (VIEGAS, 2008).

Apesar da HA primária ser de causa desconhecida, diversos organismos reconhecem a importância de fatores relacionados com o estilo de vida que contribuem fortemente para o desenvolvimento da HA, como o consumo excessivo de sódio (HEIMANN et al., 2002; GIL; LOPES, 2009), o excesso de peso, a inatividade física e o consumo de álcool, sendo o consumo de sódio referido como o principal dos fatores (AMODEO; LIMA, 1996; VIEGAS, 2008).

O sal de mesa ou sal de cozinha é um composto, quimicamente denominado de Cloreto de Sódio $(\mathrm{NaCl})$. O sal é talvez o condimento mais antigo, usado pelo homem, supondo-se que o seu aparecimento data de 2700 a.C, na China. Já o sódio é um micronutriente essencial ao organismo e constitui $40 \%$ do sal de cozinha, sendo que $10 \%$ do sódio ingerido apresenta sua origem no conteúdo natural dos alimentos e o restante é proveniente de adição (VIEGAS, 2008).

Segundo as VI Diretrizes Brasileiras de Hipertensão Arterial, vários pesquisadores concordam com a tese de que a ingestão excessiva de sal eleva a pressão arterial (CAPALONGA et al., 2010). Inúmeras são as evidências do papel do sódio na fisiopatologia da HA, tanto em animais, quanto no ser humano. A cepa de ratos Dahl sensível a sal, o modelo doca-sal, a coartação da aorta e doses não pressoras de angiotensina II em cães, são alguns exemplos, nos quais o vínculo sal-hipertensão tem sido verificado em animais (AMODEO; LIMA, 1996).

A principal função do sódio no organismo é controlar o volume do fluido extracelular e do plasma, tendo também um papel importante na condução dos impulsos nervosos, na contração muscular, na manutenção da pressão oncótica e/ou coloidosmótica e no equilíbrio ácido-base (GUYTON; HALL, 2011; MCARDLE et al., 2011). Segundo Carvalho e Mara (2010), na composição plasmática predominam os cátions de sódio $(142 \mathrm{mEql} / \mathrm{L})$, secundados pelos ânions de cloro e pequenas quantidades de proteínas e potássio $(4,2 \mathrm{mEq} / \mathrm{L})$.

A composição do líquido extracelular é rigorosamente regulada por diversos mecanismos, com destaque para a função renal, o que mantém as células banhadas por um líquido com concentração de eletrólitos e nutrientes apropriada ao seu perfeito funcionamento (GUYTON; HALL, 2011; MCARDLE et al., 2011; MARTELLI, 2013).

Quando a ingestão de sódio excede as necessidades do organismo existem mecanismos de controle que funcionam de forma a manter o normal conteúdo de sódio nos fluidos corporais. No entanto, calcula-se que existe um limite superior até ao qual se pode eliminar sódio, e uma ingestão para além deste limite, provoca um aumento no conteúdo de sódio e, consequentemente retenção de água, aumento da volemia e da pressão arterial (VIEGAS, 2008).

Se a ingestão excessiva for pontual podem não existir manifestações, no entanto, quando a ingestão é mantida por longos períodos de tempo, podem ocorrer alterações irreversíveis nos mecanismos de adaptação, provocando danos nos tecidos e falha dos mesmos, considerando-se que uma das manifestações que resulta destes danos é a HA (VIEGAS, 2008).

Amodeo e Lima (1996) relatam em seu estudo que no âmbito populacional, a ingestão de sódio pode ser um dos fatores envolvidos no aumento da pressão arterial, evidenciado com o envelhecimento. Em um amplo estudo epidemiológico internacional, o INTERSALT, concluiu-se que houve correlação direta entre quantidade de sal, habitualmente ingerida e elevação da pressão arterial com a idade, havendo aumento discreto da pressão arterial, ou mesmo ausência de elevação, em populações com reduzida ingestão de sódio.

De acordo com Olmos e Benseñor (2001), o Intersalt foi um estudo multicêntrico realizado na década de 1980 que visou a determinar o papel da ingestão de sal e de outros fatores na determinação dos níveis pressóricos de várias populações, analisando 10.079 homens e mulheres de 20 a 59 anos em 52 centros de 32 países diferentes, incluindo populações com diferentes culturas e estilos de vida.

Novos estudos nesta área vêm sendo realizados como o descrito por He et al. (2009), que realizaram um estudo controlado sobre o papel da ingestão de sal e potássio na pressão arterial. Este estudo de intervenção alimentar envolveu 1906 indivíduos, incluindo 7 dias de dieta pobre em sal (3g/dia), 7 dias de dieta rica em sal (18g/dia) e 7 dias de dieta rica em sal (18g/dia) suplementada com potássio 
(2,34 g/dia), sendo verificado que a restrição na ingestão de sal é uma das mais eficientes ferramentas terapêuticas na homeostase da pressão arterial.

Uma pesquisa realizada por Pessuto e Carvalho (1998) quanto ao uso de sal na alimentação, verificaram que $85,7 \%$ de sua amostra concordaram que este mineral interfere na pressão arterial, $8,6 \%$ não souberam responder, $4,3 \%$ acham que não há interferência e $1,4 \%$ não responderam, sendo que $40,0 \%$ dos entrevistados chegaram neste consenso por experiência própria, $25,6 \%$ por orientação médica, $5,8 \%$ receberam orientação em grupo e os demais deram outras respostas.

Veiga et al. (1993), entrevistaram 1.210 pessoas com idade entre 8 e 81 anos referente aos fatores de risco da $\mathrm{HA}$, onde $40 \%$ da amostra identificou o sal como um significativo fator de risco e $21,1 \%$ desta parcela se consideram expostos a este fator, sendo possível observar por esses dados que os entrevistados podem não ter noção de sua exposição a esse fator de risco, uma vez que no Brasil é habitual a ingestão excessiva de sódio.

Estudos demonstram a existência de relação direta entre a excreção urinária de sódio e a pressão arterial média. Elevações da pressão arterial levam ao aumento imediato da diurese e da perda renal de sódio, um fenômeno conhecido como natriurese pressórica (GONÇALVES et al., 2000; HEIMANN et al., 2002) e na vigência de consumo hidrossalino inalterado, os rins provocam a redução da volemia e do débito cardíaco, resultando no retorno e controle da pressão aos valores iniciais (HEIMANN et al., 2002; ZATS, 2002; MARTELLI, 2013).

Levando-se em consideração que mais de $95 \%$ do sódio ingerido é excretado na urina, e que a avaliação dietética apresenta muitos problemas operacionais, a excreção urinária de $24 \mathrm{~h}$ vem sendo utilizada como um marcador do consumo diário de sódio, apesar da grande variabilidade intraindividual. Assim sendo, interpretações clínicas e fisiológicas baseadas numa única avaliação devem ser cautelosas (MOLINA et al., 2003).

Segundo Heimann et al. (2002), a presença de qualquer nefropatia que comprometa a natriurese pressórica deixa a pressão arterial mais ou exclusivamente dependente da resistência periférica figura 2. A HA é altamente prevalente em todos os casos de doença renal crônica sendo um fator que contribui para a aceleração do declínio da função renal.

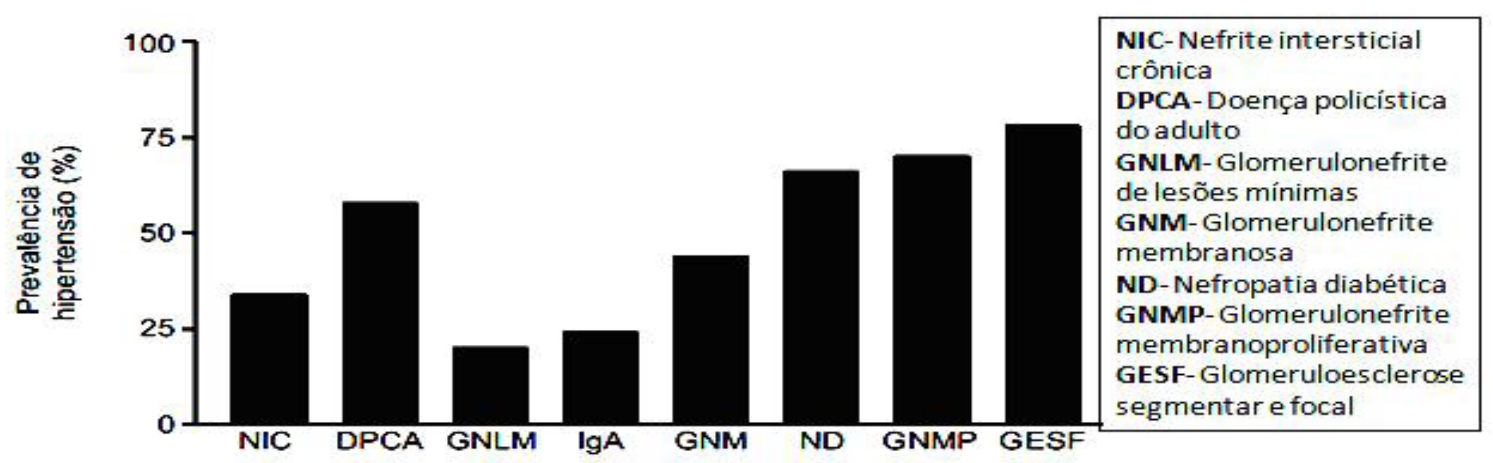

Figura 2. Prevalência de HA em doenças renais parenquimatosas crônicas. Extraído e Modificado de Heimann (2002).

Segundo Martelli (2013), o rim e a pressão arterial interagem de maneira íntima e complexa e a grande maioria dos pacientes com doença renal desenvolve ou agrava os quadros de HA à medida que a função renal diminui assim como no manuseio renal de sódio implicando na patogênese da HA primária humana.

Apesar de consolidada a relação entre HA e os fatores nutricionais, ainda não são bem esclarecidos os mecanismos de atuação destes sobre a elevação da pressão arterial. São conhecidos, no entanto, os efeitos de uma dieta saudável, rica em frutas e vegetais e pobre em gordura sobre o comportamento dos níveis pressóricos (SACKS et al., 2001).

Por outro lado, Sarno (2010) relata que a resposta da pressão arterial frente a modificações no 
consumo de sódio não é homogenia na população, existindo indivíduos que apresentam tendências diferentes de oscilação da pressão arterial frente a reduções ou suplementações de sal, fenômeno este conhecido como sensibilidade ao sal.

As questões relacionadas à sensibilidade ao sódio parecem ser de difícil resolução quando são estudadas em seres humanos (MOLINA et al., 2003). Efetivamente, alguns indivíduos excretam maiores quantidades de sódio sem um aumento na pressão arterial e outros não (COWLEY, 1997).

A resposta fisiológica a um aumento na ingestão de sódio resultaria na redução da atividade do sistema renina-angiotensina-aldosterona (MARTELLI, 2010) e um aumento na liberação do peptídio natriurético atrial, sendo que cada um desses sistemas interage com outros sistemas e entre si, além de atuarem na redução da atividade simpática direcionada para os rins. As diferenças genéticas responsáveis por estas diferentes respostas do organismo ainda não são bem conhecidas em humanos, sendo difícil estabelecer, a priori, quem estaria no primeiro ou no segundo grupo, quando é estudado grande número de indivíduos na população, principalmente quando ela é heterogênea do ponto de vista racial (MOLINA et al., 2003).

Uma alimentação mais pobre em frutas e hortaliças e baseada em alimentos industrializados, mais rica em gordura e sal, parece ser preditora de agravos à saúde, particularmente associada aos níveis pressóricos e o alto consumo de sódio também pode estar relacionado à maior ingestão de alimentos preparados com temperos prontos, bastante acessíveis às classes socioeconômicas menos favorecidas. (MOLINA et al., 2003).

O DASH (Dieta Adequada para Controlar a Hipertensão) foi um estudo multicêntrico que mostrou que uma dieta rica em frutas, hortaliças, laticínios desnatados e com baixo teor de gordura total e saturada diminui a pressão sistólica em média 6 a 11 milímetros de mercúrio (mmHg) (MCARDLE et al., 2011; OLMOS; BENSEÑOR, 2001).

Esta dieta é usada tanto na prevenção quanto no controle da HA (OLMOS; BENSEÑOR, 2001). Cada $2 \mathrm{mmHg}$ de redução na pressão sistólica acarreta uma queda de 5\% no risco de doenças cardíacas e de 8\% no risco de acidente vascular cerebral (MCARDLE et al., 2011).

Cappuccio (1997) ressalta a necessidade de redução moderada de sal na dieta e aumento de alimentos ricos em potássio não apenas como um primeiro passo no tratamento de indivíduos com HA, mas, sobretudo, como medida preventiva para a redução da prevalência da HA e suas complicações na população.

Segundo a ANVISA (2012), em abril de 2011, o Ministério da Saúde assinou termo de compromisso com a Associação Brasileira das Indústrias de Alimentação (ABIA), a Associação Brasileira das Indústrias de Massas Alimentícias (ABIMA), a Associação Brasileira da Indústria de trigo (ABITRIGO) e a Associação Brasileira da Indústria de Panificação e Confeitaria (ABIP) com a finalidade de estabelecer metas nacionais para redução do teor de sódio em macarrão instantâneo, pão de forma e bisnaguinhas e em dezembro de 2011 assinou outro termo com essas mesmas associações ampliando a gama de produtos processados para pão francês, bolos prontos sem recheio, bolos prontos recheados, rocambole, bolo aerado, bolo cremoso, salgadinhos de milho, batatas fritas, batatas palhas, maionese, biscoito doce (Maizena e Maria), biscoito salgado (Cream Cracker, água e sal) e biscoito doce recheado. Em agosto de 2012, assinou um terceiro termo de compromisso com as mesmas associações para os cereais matinais, a margarina vegetal, os caldos líquidos e caldos em gel, os caldos em pós e caldos em cubo, os temperos em pasta, os temperos para arroz e demais temperos.

Como recomendações da ANVISA (2012), o consumidor deve observar a rotulagem nutricional para identificar os alimentos embalados com menor teor de sódio e proteger a sua saúde e de sua família, devido a existência de uma grande variação na quantidade de sódio entre alimentos de uma mesma categoria ou similares figura 3.

Atualmente, em nosso país, a prevenção de doenças e a promoção à saúde são temáticas ainda pouco exploradas. No entanto, acredita-se que esse quadro possa ser revertido por meio da união de esforços entre os diferentes segmentos da sociedade.

Se instituições de âmbito da saúde, educação política e econômica unissem esforços voltados para prevenção e promoção da saúde quanto aos fatores de risco de doenças crônico-degenerativas, certamente a curto prazo essa lacuna poderia ser preenchida, atendendo à melhoria da qualidade de vida do homem brasileiro. 


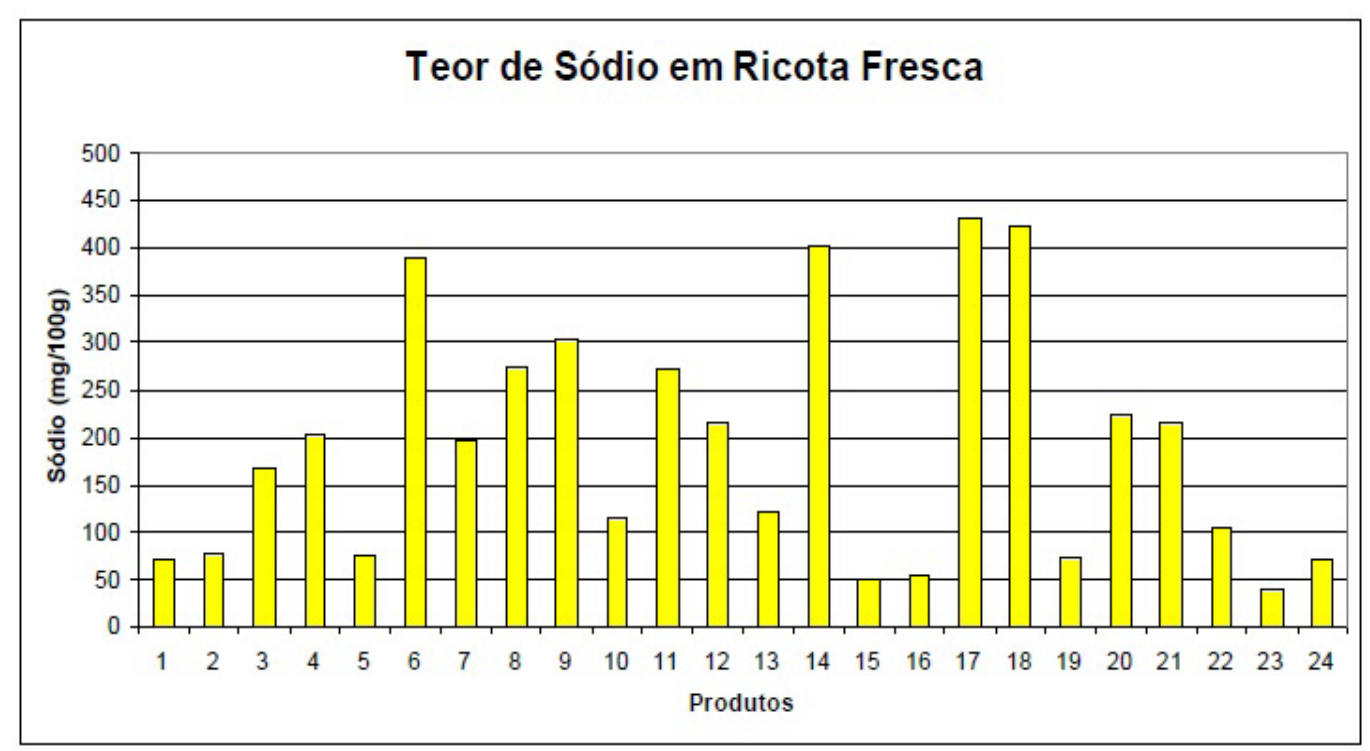

Figura 3. Resultados das análises de sódio em 24 marcas diferentes de ricota fresca. Teor médio de sódio = 191mg/100g com variação de 41mg até 432mg. Extraído ANVISA, 2012.

\section{CONCLUSÃO}

A hipertensão arterial continua sendo uma das mais importantes morbidades nos adultos, estando direta ou indiretamente relacionada à principal causa de morte no País.

A redução do consumo de sódio pela população brasileira, assume posição de destaque dentro da agenda da saúde no Brasil, mediante diminuição do sal adicionado durante o preparo e o consumo de alimentos e do sódio presente nos alimentos industrializados, trabalhando eixos de comunicação, reformulação, monitoramento e regulação.

Apesar de consolidada a relação entre hipertensão arterial e os fatores nutricionais, ainda não são bem esclarecidos os mecanismos de atuação destes sobre a elevação da pressão arterial, necessitando de novos estudos.

A mudança do estilo de vida é uma atitude que deve ser estimulada em todos os pacientes hipertensos ou não, durante toda a vida, através de modificações dietético-comportamentais, que contribuirão, em muito, para o melhor controle da pressão arterial.

Acredita-se que a educação dos indivíduos seja o melhor caminho para o alcance de uma redução dos casos de hipertensão arterial, não sendo apenas uma transmissão de conteúdos referentes à patologia e ao tratamento, mas sim que se promova a adaptação dos pacientes ao tratamento. Para se chegar a essa adaptação, é preciso que os indivíduos estejam motivados para que tais mudanças ocorram e, também, para que assimilem os conhecimentos que poderão melhorar a qualidade de vida dos mesmos.

\section{REFERÊNCIAS}

AMODEO, C.; LIMA, N.K.C. Tratamento não medicamentoso da hipertensão arterial. Medicina Ribeirão Preto, v. 29, p.239-243, abr./set., 1996.

AGENCIA NACIONAL DE VIGILÂNCIA SANITÁRIA - ANVISA. INFORME TÉCNICO N. 50/2012 TEOR DE SÓDIO DOS ALIMENTOS PROCESSADOS. Disponivel em < http://portal.anvisa.gov.br/wps/ $\mathrm{wcm} /$ connect/856c37804d19e24d9d7aff4031a95fac/INFORME+T\%C3\%89CNICO+2012-+OUTUBRO. pdf?MOD=AJPERES $>$ Acesso em 14 Nov 2013.

BIBBINS-DOMINGO K.; CHERTOW, G.M.; COXSON, P.G.; MORAN, A.; LIGHTWOOD, J.M.; 
PLETCHER, M.J. et al. Projected effect of dietary salt reductions on future cardiovascular disease. N Engl J Med. v. 362, n. 7, p.590-9, 2010.

BROWN, I.J.; TZOULAKI, I.; CANDEIAS, V.; ELLIOTT, P. Salt intakes around the world: implications for public health. Int J Epidemiol. v. 38, n. 3, p.791-813, 2009.

Brasil, Ministério da Saúde, Secretaria de Vigilância à Saúde, Secretaria de Gestão Estratégica e Participativa. Vigitel Brasil 2010: vigilância de fatores de risco e proteção para doenças crônicas por inquérito telefônico. Brasília: Ministério da Saúde; 2011. Disponível em: <http://bvsms.saude.gov.br/bvs/publicacoes/vigitel 2010.pdf> Acessado em novembro de 2013.

CAPALONGA, R.; et al. Avaliação da quantidade de sal oferecida no almoço dos funcionários de um Hospital Público de Porto Alegre. Revista HCPA. v. 30, n. 3, p.204-20, 2010.

CAPPUCCIO, F.P. Dietary changes and their effect on blood pressure: what lesson should we learn? J Hum Hypertens, v. 11, p. 765-6, 1997.

CARVALHO, T.; MARA, L. S. Hidratação e Nutrição no Esporte. Rev Bras Med Esporte. v. 16, n. 2, Mar/ Abr, 2010.

COWLEY, A.W. Genetic and nongenetic determinants of salt sensibility and blood pressure. Am J Clin Nutr. v. 6, (Suppl), p.587-93, 1997.

DRAGER, F.; KRIEGER, J.E. A genética das síndromes hipertensivas endócrinas. Arq. Bras. Endocrinol. Metab. v. 48, n. 5, p.659-665, 2004.

GIL, J.S.; LOPES, H.F. Fisiopatologia da pré-hipertensão. Rev Bras Hipertens v. 6, n. 2, p.87-91, 2009.

GONÇALVES, A.R.R.; ZATZ, R.; HEIMANN, J.C. O papel do rim no controle da pressão arterial. Hipertensão, v. 3, n. 1, p.6-14, 2000.

GUYTON, A. C.; HALL, J.E. Tratado de fisiologia Médica. Rio de Janeiro: Elsevie; 2011.

HE, F.J.; MACGREGOR, G.A. A comprehensive review on salt and health and current experience of worldwide salt reduction programmes. J Hum Hypertens. v. 23, n. 6, p.363-84, 2009.

HE, J.; GU, D.; CHEN, J. et al. Gender difference in blood pressure responses to dietary sodium intervention in the GenSalt study. J Hypertens. v. 27, n. 1, p.48-54, 2009.

HEIMANN, J.C.; VIDONHO JUNIOR, A.F.; RUIVO, G.F. Mecanismos hipertensores em indivíduos portadores de nefropatia crônica. Rev Bras Hipertens, v. 9, n. 2, p.135-40, 2002.

MARTELLI, A. Sistema Renal e sua Influência no Controle em Longo Prazo da Pressão Arterial. UNOPAR Cient Ciênc Biol Saúde, v. 15, n. 1, 2013.

MARTELLI, A. Sistema Renina Angiotensina Aldosterona e Homeostase Cardiovascular. UNOPAR Cient Ciênc Biol Saúde, v. 12, n. 4, 2010.

MCARDLE, W. D.; KATCH, F. I.; KATCH, V. L. Fisiologia do Exercício: Energia, Nutrição, Energia e Desempenho Humano. $7^{\mathrm{a}}$ ed. Rio de Janeiro, RJ: Guanabara Koogan, 2011.

MIRANDA, R.D.; PERROTTI, T.C.; BELLINAZZI, V.R.; NÓBREGA, T.M.; CENDOROGLO, M.S.; TOMIOLO, NETO J. Hipertensão arterial no idoso: peculiaridades na fisiopatologia, no diagnostico e no 
tratamento. Rev Bras Hipertens. v. 9, n. 3, p.293-300, 2002.

MOLINA, M.C.B.; CUNHAB, R.S.; HERKENHOFFB, L.F.; MILLB, J.G. Hipertensão e consumo de sal. Rev Saúde Pública, v. 37, n. 6, p.743-50, 2003.

NICOLAIDIS, S. Prenatal imprinting of postnatal specific appetites and feeding behavior. Metabolism. v. 57, Suppl. 2, p.22-6, 2008.

NILSON, E.A.F.; JAIME, P.C.; RESENDE, D.O. Iniciativas desenvolvidas no Brasil para a redução do teor de sódio em alimentos processados. Rev Panam Salud Publica. v. 34, n. 4, p.287-92, 2012.

OLMOS, R.D.; BENSEÑOR, I.M. Dietas e hipertensão arterial: Intersalt e estudo DASH. Rev Bras Hipertens v. 8, n. 2, 2001.

PESSUTO, J.; CARVALHO, E.C. Fatores de risco em indivíduos com hipertensão arterial. Rev. latinoam. Enfermagem, Ribeirão Preto, v. 6, n. 1, p. 33-39, janeiro 1998.

RIELLA, M.C.; MARTINS, C. Nutrição e o rim. Rio de Janeiro: Guanabara Koogan; 2001.

SACKS, F.M.; SVETKEY, L.P.; VOLLMER, W.M.; APPEL, L.J.; BRAY, G.A.; HARSHA, D. et al. Effects on blood pressure of reduced dietary sodium and the Dietary Approaches to Stop Hypertension (DASH) diet. N Engl J Med, v. 344, p.3-10, 2001.

SALAS, C. K. T. S. et al. Teores de sódio e lipídios em refeições almoço consumidas por trabalhadores de uma empresa do município de Suzano, SP. Rev. Nutrição v. 22, n. 3, p. 331-339, 2009.

SARNO, F. Estimativas do consumo de sódio no Brasil, revisão dos benefícios relacionados à limitação do consumo deste nutriente na Síndrome Metabólica e avaliação de impacto de intervenção no local de trabalho. São Paulo. [Tese de Doutorado. Faculdade de Saúde Publica da USP], 2010.

VEIGA, E.V.; ROBAZZI, M.L.C.C.; NOGUEIRA, M.S.; TAKAKURA, M.S.; HAYASHIDA, M. Estudo dos fatores de risco da hipertensão arterial: conhecimento e exposição. Rev Soc Cardiol Estado de São Paulo, n.6 (supl A), p.1-5, 1993.

VIEGAS, C. Sal e doença cardiovascular. Revista Factores de Risco, n. 10, p.12-18, Jul-Set, 2008.

WAITZBERG, D.L. Nutrição oral, enteral e parenteral na prática clínica. 3ª ed. São Paulo: Atheneu; 2001.

WANG, G.; LABARTHE, D. The cost-effectiveness of interventions designed to reduce sodium intake. J Hypertens. v. 29, n. 9, p.1693-9, 2011.

WEBSTER, J.L.; DUNFORD, E.K.; HAWKES, C.; NEAL, B.C. Salt reduction initiatives around the world. J Hypertens. v. 29, n. 6, p.1043-50, 2011.

ZATS, R. Fisiopatologia renal. São Paulo: Atheneu; 2002. 